\title{
Theory and Conceptual Design of a High-Power Highly Efficient Magnicon at 10 and $20 \mathrm{GHz}$
}

\author{
WALLACE M. MANHEIMER, SENIOR MEMBER, IEEE
}

\begin{abstract}
It is shown that magnicons can be scaled up to high frequency ( $X$-band and above) at high power and efficiency. Magnicons have an advantage over other slow wave devices in that the structure sizes are comparable to or larger than a wavelength, so RF breakdown should be less of a problem. However, they require higher voltage. The magnicon has the promise of considerably enhancing the technology of efficient high-power high-frequency RF sources.
\end{abstract}

\section{INTRODUCTION}

$\mathrm{T}$ HIS PAPER describes a theory and conceptual design for a magnicon at frequencies of 10 and $20 \mathrm{GHz}$. The magnicon was first developed in the Soviet Union [1] where it was billed as an improved version of the gyrocon; an RF source also developed there [2], [3]. Although gyrocons and magnicons were initially developed for frequencies below $1 \mathrm{GHz}$, a recent Soviet presentation at an international accelerator conference [4] has indicated that gyrocons have been developed at powers of $60 \mathrm{MW}$, at a pulselength of $700 \mathrm{~ns}$ and at a frequency of $7 \mathrm{GHz}$ with $25 \%$ efficiencies. The drive power was at $3.5 \mathrm{GHz}$. At present, this exceeds the performance of klystrons.

As we will see, magnicons appear to have even greater potential at high frequency in terms of both power and efficiency than gyrocons do. Magnicons also have the advantage that the cavity sizes are comparable to, or even larger than, a wavelength. This is a tremendous advantage over klystrons where, at $X$-band and above, the sizes get very small and RF induced breakdown in the cavities and gaps becomes very difficult to avoid. However, counterbalancing the advantage of large size cavities and gaps is the fact that high voltage and rather low pervience is required. The reason is that in both the gyrocon and magnicon the electron beam travels a certain distance without any focusing, meaning the electron energy must be large so that beam self-forces are reduced. In the example discussed here we take a voltage of $1 \mathrm{MV}$, which is about the maximum that can be achieved with a conventional pulse-line modulator and voltage step-up transformer [5]

The magnicon would appear to have an application wherever very efficient phase-locked sources of highpower high-frequency radiation are needed. Such applications include particle acceleration [6], [7], space power

Manuscript received September 27, 1989; revised January 25, 1990 This work was supported by the Office of Naval Research.

W. M. Manheimer is with the Plasma Physics Division, Naval Research Laboratory. Washington. DC 20375.

IEEE Log Number 9035696 beaming [8], large phased array radars [9], and interactive discrimination for strategic defense [10]. The principal application to be considered here is as a drive for a $\mathrm{TeV}$ linear collider.

The task of building the next generation of $\mathrm{TeV}$ linear colliders will require the RF sources to power the $\mathrm{TeV}$ linear colliders in a way that cannot be met with existing technology. The requirement is generally considered to be approximately $500 \mathrm{MW} / \mathrm{m}$ of $\mathrm{RF}$ power with a pulselength of $50 \mathrm{~ns}$ at a frequency between 10 and $20 \mathrm{GHz}$ [6], [7]. Because the power draw of the klystrons will be a large fraction of the operating cost of the accelerator, there is a premium on high efficiency sources. There are two basic approaches to achieving this goal. The first is to generate lower power in a long RF pulse, perhaps for $1 \mu \mathrm{s}$, and then compress this pulse to the shorter time while simultaneously multiplying the power by the compression ratio. This is the basic approach currently used on the SLAC at present, where $67 \mathrm{MW}$ of power is compressed to about $150 \mathrm{MW}$ by the SLED pulse compression scheme. The other approach is to directly generate power at the $500-\mathrm{MW}$ level with a $50-n$ sulselength. This latter scheme obviously requires an intensepulsed electron beam, having a gigawatt power level. In order to explore the various options, $X$-band klystrons are being developed at SLAC, LLNL, and LBL [6]. So far, in a conventional klystron with a $1-\mu$ s pulse, about 24 MW at $8 \mathrm{GHz}$ has been achieved with $40 \%$ efficiency. $X$ band gyroklystrons are being developed at the University of Maryland [11]. This paper contends that magnicons, and possibly gyrocons also are potential sources to power $\mathrm{TeV}$ colliders. We will first review previous results in gyrocons and magnicons and then give a conceptual design for a 50-70-MW magnicon at 10 and $20 \mathrm{GHz}$.

\section{Review of Previous Work on Gyrocons and MAGNICONS}

In this section we revie previous work on gyrocons and magnicons. The major portion of this paper has been carried out in the Soviet Union [1]-[3], although there has been a small effort on gyrocons at Los Alamos as well [12]-[14]. Generally, the Soviet effort has been much more successful. The gyrocon was invented by Budker [2] and much of the work on it is summarized in his review article [3]. The magnicon is a more recent invention and is a modification of the gyrocon concept. Up until very recently, gyrocons and magnicons have claimed that their 
best performance would be at very low frequency. The Novosibirsk gyrocons were designed and built at frequencies of 180 and $430 \mathrm{MHz}$. The Los Alamos gyrocon was designed at a frequency of $450 \mathrm{MHz}$. The Novosibirsk magnicon was built at a frequency of $915 \mathrm{MHz}$. These frequencies are too low to be of interest as sources to power TeV linear supercolliders. What is extremely fascinating is that at the Workshop on Linear Colliders in Capri in June 1988, the Soviets under Balakin [4] presented dramatic new results on gyrocons. A power of 60 $\mathrm{MW}$ and an efficiency of $25 \%$ at a frequency of $7 \mathrm{GHz}$ was claimed, with a $0.7-\mu$ s pulse at a $1-2 \mathrm{~Hz}$ repetition rate. The device was a five-cavity frequency-doubled amplifier with a gain of $60 \mathrm{~dB}$. The drive frequency was 3.5 $\mathrm{GHz}$. Our conclusion is that magnicons have even greater potential at the higher frequencies.

We now discuss the gyrocon and briefly review some of the previous experimental work. Both the klystron and the gyrotron (or gyroklystron) are limited by the fact that the beam is uniformly distributed in phase at the entrance to the first cavity. In the case of the klystron, since it is a linear device, the phase is simply the entrance time to the first cavity relative to the phase of the wave. In the case of the gyrotron, since it is a crossed-field device, the phase is the azimuthal angle in momentum space relative to the phase of the wave in the cavity. In either case, this input phase is distributed uniformly over $2 \pi$. This means that at the output cavity, some of the electrons will be phased wrong and will take energy from the radiation field in the cavity even though most electrons give their energy to the field. The gyrocon is an extremely ingenious way to avoid this difficulty. In an input cavity (called a scanner cavity by Budker), the beam is given a transverse displacement by a rotating mode launched in that cavity. In the Soviet experiments, a rotating $\mathrm{TM}_{110}$ mode was used. Since the mode is rotating, the transverse velocity at the exit of the input cavity also rotates at the drive frequency. Following the input cavity, there is a drift space. The beam enters this drift space with a particular polar angle $\alpha=v_{t} / v_{\text {z }}$ which does not vary in time, and with an azimuthal angle $\phi=\arctan v_{x} / v_{y}$ which varies in time at the drive frequency. Here $z$ is the axis of the device and $v_{t}=\left(v_{x}^{2}+\right.$ $\left.v_{y}^{2}\right)^{1 / 2}$. A schematic of the input cavity, with the fields launched by two coupling apertures separated by $90^{\circ}$ geometrically and excited with a $90^{\circ}$ phase shift electronically, is shown in Fig. 1. The polar angle $\alpha$ depends on the strength of the field in the input cavity. If the input $\mathrm{RF}$ source is limited in power, it is possible to increase $\alpha$ by adding additional passive deflection cavities just as in the case of a klystron or a gyroklystron. Since the initial NRL magnicon design will rely on a high-power driver, it will have only a single deflection cavity before the drift space. The initial experiment is designed for a gain of only $10-17 \mathrm{~dB}$. However, total gain is ultimately an important parameter for an accelerator. This is particularly true where it would be desirable to have a single oscillator control the entire accelerator. In this case, phase shifting could be done at very low power. To achieve high gain,

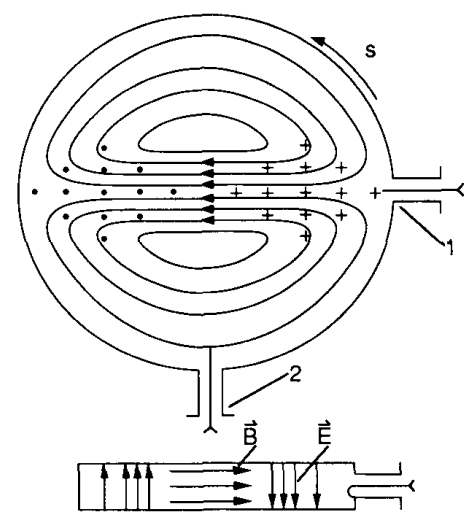

Fig. 1. Schematic of the input cavity with a rotating $\mathbf{T M}_{110}$ mode launched.

additional passive cavities could be used, as in the case of a klystron or gyroklystron. Karliner's magnicon experiment had two input cavities and achieved a gain of 30 $\mathrm{dB}$. Balakin's gyrocon experiment had 3-5 input cavities and achieved a gain of $60 \mathrm{~dB}$. Designs with additional bunching cavities are straightforward and will be discussed later.

After the beam traverses the drift space, it enters the output resonator through a azimuthal slot. The azimuthal angle, at which particular electrons enters, varies linearly in time with an angular velocity $\omega$ (the drive frequency). The entering electrons then excite a rotating wave in the output cavity which is resonant; that is, it also has an angular velocity $\omega$. Since all electrons now see the same phase of the wave, each electron can now give almost all of is energy to the fields in the cavity. Thus extremely high efficiency (approaching 100\%) is theoretically possible.

Budker points out that one of the most difficult problems in realizing the potential of the gyrocon is the problem of beam focusing. In the drift region, the beam will spread out due to electrostatic repulsion (partially cancelled by self-pinching at high energy) combined with its emittance. Budker points out that it is very important to use a high energy beam so as to cancel out the electrostatic field as much as possible. As we will see, a figure of merit for the self-fields is the quantity $I / \beta^{3} \gamma^{3}$. Small values of this parameter imply weak electrostatic defocusing. This is the quantity which appears in the paraxial ray equation for the beam. This will be an important quantity to keep track of when comparing the measured performance of different gyrocons or magnicons. A schematic of the beam entering the output cavity is shown in Fig. 2. Note that the focusing of the beam in its radial dimension is extremely critical, but that focusing in the azimuthal direction is not. Another difficulty with the gyrocon, not pointed out by Budker but by Los Alamos [15], is the coaxial nature of the entrance slot to the output cavity. Since the slot has no minimum cutoff frequencymode conversion, a radiation leakage can arise which then propagates back toward the electron gun and degrades the beam quality in the drift space. 


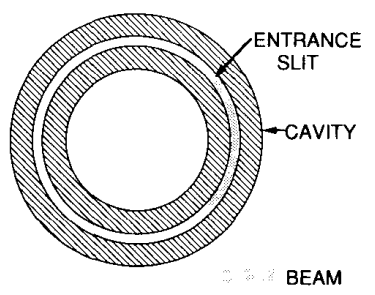

Fig. 2. Input to the output cavity of the gyrocon.

The gyrocon has the same difficulty in scaling to high frequency as the klystron does. The deceleration of the electron beam in the output cavity must take place in half a wavelength or less. Thus the RF electric field strength must be sufficient to show the electron down to zero velocity in less than half a wavelength. If the tolerable electric field at the surface of the conductor is given by $E_{\mathrm{tol}}$, then an approximate minimum wavelength of the gyrocon is given by $\lambda_{\min }=2(\gamma-1) m c^{2} / E_{\mathrm{tol}}$. A similar but more precise relation based on constants of the motion is given by Budker [3].

Finally, let us note that the gyrocon, or at least the gyrocon with the single deflection cavity, is an inherently nonlinear device. There is no range of linear amplification. For instance, if the input power is not at the right value, the deflected electrons will miss the slot in the output cavity. The deflection cavity simply sets up the beam so that the electrons are properly phased with respect to the wave in the output cavity. The inherent nonlinear nature of the gyrocon and the magnicon has important implications on their performance. The power and efficiency can be extremely large. However, other figures of merit, particularly the bandwidth, are sacrificed. This is not a problem for the Department of Energy (DOE) application. however, because the source to power a $\mathrm{TeV}$ linear supercollider has no requirements for bandwidth. Nevertheless, it is important to note that to achieve optimum performance, the driver must have very precise amplitude and frequency control.

Experimental development of gyrocons and magnicons has taken place in Novosibirsk and Los Alamos [1]-[4], [12]-[14]. A schematic of the Novosibirsk pulsed gyrocon is shown in Fig. 3. The results of various gyrocon experiments are summarized on Table I. The last two rows are magnicons, while the gyrocon information is on the rows above. Notice that the low frequency Novosibirsk gyrocons had a relatively easy time in meeting the physics objectives with regard to amplification and efficiency, but had more difficulty regarding the technological specifications with regard to power and pulselength. The $7-\mathrm{GHz}$ gyrocon result is included in the table only as an acheived value since the design was not published. Notice that the gyrocon, which did not meet the physics specifications that the Soviets have been able to achieve, was the Los Alamos gyrocon. Its efficiency was only $23 \%$. However, this device in a sense attempted more than the Soviet experiments in that it utilized a much higher pervience beam.

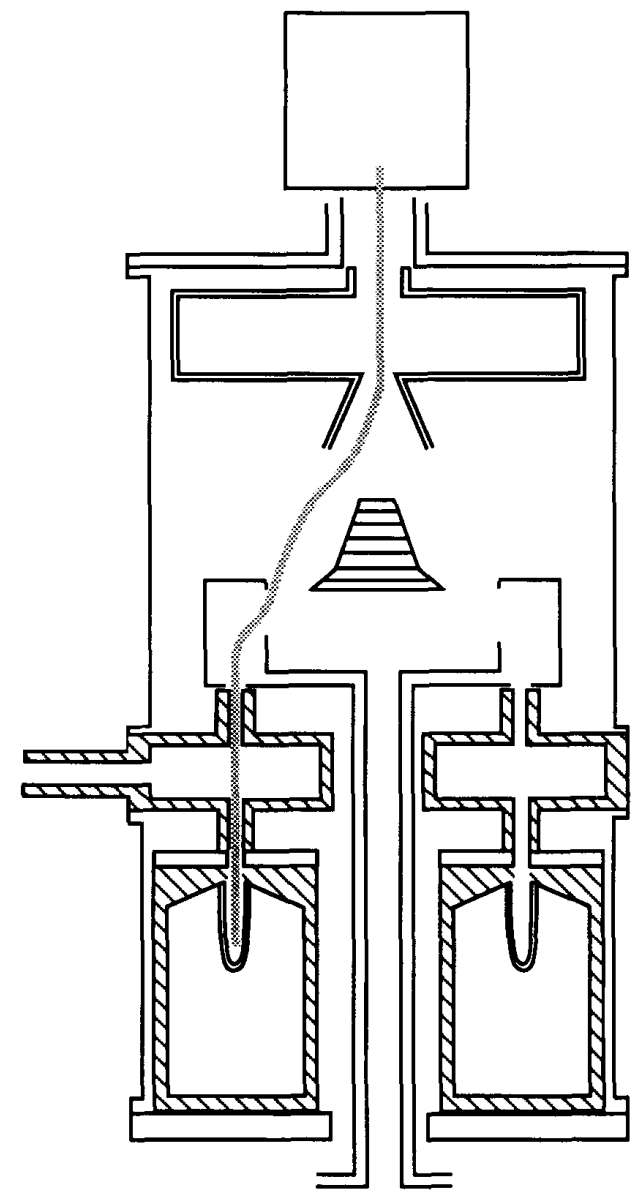

Fig. 3. Conceptual design of the Novosibirsk gyrocon. Achieved and proposed results of gyrocons and magnicons.

Notice that the space charge parameter $I / \beta^{3} \gamma^{3}$ is about one order of magnitude larger than in the Soviet experiments.

We now turn to a discussion of the magnicon. As we have seen, the gyrocon is limited in at least three fundamental ways. First, since the deceleration occurs in half a wavelength the minimum wavelength is limited by the electric field that the cavity can withstand. Second, the annular entrance slit in the output cavity implies a difficult beam transport problem, particularly as the current and therefore the power increase. Third, because of the annular entrance slit, radiation can leak out of the output cavity into the drift space, reducing efficiency and interfering with beam transport. The magnicon can cure all of these difficulties while retaining all of the advantages of high power and high efficiency of a gyrocon. A brief summary of the physics of the magnicon is given here, followed by a detailed discussion in the next section.

The design of a magnicon does not differ appreciably from that of a gyrocon up to the entrance of the second cavity; that is, each exploits a periodic deflection of the beam in the bunching cavity and drift space. However, 
TABLE I

Proposed) (LfFt) and Achieved (Right) Results of Gyrocons and

MAGNICONS

\begin{tabular}{|c|c|c|c|c|c|c|c|c|c|c|c|c|c|}
\hline Device & $\mathrm{v}$ & I & $\eta$ & f & $\tau$ & Gain $\mathrm{db}$ & $v$ & I & $\eta$ & f & $\tau$ & G & $I / \beta^{3} \gamma^{3}(A)$ \\
\hline N.G. Initial & & & & & & & $320 \mathrm{~K}$ & 2 & $90 \%$ & $430 \mathrm{M}$ & $20 \mu$ & & 1 \\
\hline N.G, cw & $500 \mathrm{~K}$ & 12 & $80 \%$ & $181 \mathrm{M}$ & $\mathrm{CW}$ & $23 \mathrm{db}$ & $270 \mathrm{~K}$ & 2.2 & $80 \%$ & $181 \mathrm{M}$ & $\mathrm{CW}$ & & 1.5 \\
\hline N.G. pulsed & $2 \mathrm{M}$ & 100 & $80 \%$ & $430 \mathrm{M}$ & $10 \mu$ & $25 \mathrm{db}$ & $1.4 \mathrm{M}$ & 45 & $80 \%$ & $430 \mathrm{M}$ & 64 & 24 & 0.8 \\
\hline N.G. Balakin & & & & & & & $1.2 \mathrm{M}$ & 200 & $25 \%$ & $7 \mathrm{G}$ & $0.7_{\mu}$ & 60 & 5 \\
\hline LASL G & $86 \mathrm{~K}$ & 9 & $93 \%$ & $450 \mathrm{M}$ & $1 \mathrm{msed}$ & $22 \mathrm{db}$ & $82 K$ & 8 & $23 \%$ & $450 \mathrm{M}$ & $50 \mu$ & & 60 \\
\hline N.M. & & & & & & & $320 \mathrm{~K}$ & 12 & $73 \%$ & $915 \mathrm{M}$ & $50 \mu$ & 30 & 6 \\
\hline NRL M. & $1 \mathrm{M}$ & 100 & $70 \%$ & ${ }_{20}^{10} \mathrm{G}$ & $1 \mu$ & $17 \mathrm{db}$ & & & & & & & $4^{*}$ \\
\hline $\begin{array}{l}\text { N-Novosibirs } \\
\text { G-Gyrocon / } \\
\text { M-Magnicon }\end{array}$ & 14 & & & & & & ${ }^{*}$-De & & & & & & \\
\hline
\end{tabular}

unlike in the gyrocon, the output cavity of the magnicon is magnetized. After the beam is deflected in the input cavity, it is allowed to drift off the axis just one Larmor diameter. At this point, the beam goes through a sharp magnetic step, ideally a half cusp, so that most of its energy resides in motion that is perpendicular to the magnetic field. It is this perpendicular energy which is available to drive the RF fields in the cavity because in the magnicon, unlike in the gyrocon, the beam loses its $\mathrm{ki}$ netic energy in a cyclotron resonant process. Karliner [1] claims that the problem of beam transport is eliminated in the magnicon. Our preliminary studies show that the design of the magnetic circuit appears to be one of the most difficult aspects of the magnicon design. However, we still estimate that it should be possible to come up with a magnetic field configuration which will yield $80 \%$ of the energy in the transverse motion of the electrons. As in the case of gyrocon, all particles experience the same fields in the output cavity so that the interaction efficiency can be very high. Thus the maximum interaction efficiency will be about $80 \%$. Moreover, the spent beam will be nearly monoenergetic and will have almost all of its energy in the axial direction. Thus a depressed collector should be able to recover a large fraction of the spent beam energy in order to further increase the efficiency.

The advantage of a cyclotron interaction, rather than a linear interaction, is that there is no longer a constraint that the beam lose all of its energy in half a wavelength. There is virtually no constraint at all on length. One simply designs the cavity length so that the beam loses its free energy with that field over that length at whatever maximum field the cavity walls can sustain. This advantage is similar to the advantage that a gyrotron or gyroklystron has over a klystron, as the frequency increases. In fact, the magnicon looks almost like a phase-locked gyrotron, but one for which the bunching is a delta function in the input phase angle. While the efficiency is high, the magnicon is not a peniotron. In the peniotron, there is no bunching and the main effect of the interaction in- volves a drift of the guiding centers. The magnicon has no guiding center drift, but maximizes the phase bunching.

Furthermore, the beam enters the output cavity near the center and the entrance is a circular hole of somewhat more than one Larmor diameter in radius (typically about one quarter of the cavity radius). Hence, there is no difficulty in guiding the beam to the cavity input. Also, the output cavity can now be designed so that mode leakage at the beam entrance aperture cannot occur, since the entrance is no longer in annular slot. The largest difficulty appears to be in the design of the magnetic field near the input to the second cavity. For this problem, there are commercially available computer codes, which can be used to design complicated magnetic circuits with or without the use of magnetically permeable materials.

We also note that guiding and focusing the beam are the most difficult aspects of both the gyrocon and magnicon, implying a great advantage to a high voltage operation. If the parameters of the device optimize at a maximum value of $I / \beta^{3} \gamma^{3}$, this implies that the power of the device scales as

$$
P \sim\left(\mathrm{eV} / m c^{2}\right)\left[\frac{2 \mathrm{eV}}{m c^{2}}+\left(\frac{\mathrm{eV}}{\mathrm{mc}^{2}}\right)^{2}\right]^{3 / 2} .
$$

To obtain (1) we simply solve for $I$ in terms of $\mathrm{V}$, assuming that $I$ is proportional to $\beta^{3} \gamma^{3}$. Thus while either highor low-voltage operation is possible in the high-voltage limit, the power scales as $V$ [4].

Thus it seems that the magnicon is a promising option for powering large linear $\mathrm{TeV}$ supercolliders at a power level of nearly $100 \mathrm{MW}$, with a $1-\mu \mathrm{s}$ pulselength and an efficiency of greater than $50 \%$. Based on our analysis, the magnicon is a significant improvement over the gyrocon for high-frequency applications and should be capable of achieving the power and efficiency required for DOE accelerator applications. The last two rows of Table I show the performance of the Novosibirsk magnicon and the design values of the proposed NRL magnicon. 
III. Conceptual Design of the NRL 10-20 GHz, 50-70 MW MAGNiCON

This section discusses the physics of high frequency magnicons by specifying a conceptual point design for an $X$-band magnicon with a power of about 50-70 MW and an efficiency of $50-70 \%$. An alternate design at the harmonic at $20 \mathrm{GHz}$ will also be presented. This magnicon would be powered by the new NRL long-pulse electronbeam accelerator at a voltage of $1 \mathrm{MeV}$ and a current of $100 \mathrm{~A}$ and a time in excess of $1 \mu \mathrm{s}$. This part on the technical design will be broken into separate sections dealing with the cathode, the beam acceleration and transport system, the deflection in the input cavity, the design of the input cavity, the orbits in the output cavity, the design of the output cavity, harmonic operation, and multicavity (high amplification) operation.

\section{A. The Cathode}

The parameter which we use to measure the characteristics of a cathode is the brightness

$$
b=I / \pi^{2} \epsilon^{2}
$$

where $\epsilon$ is the beam emittance. In units of kiloamperes per $\pi^{2} \mathrm{~cm}^{2} \mathrm{rad}^{2}$, the brightness of a SLAC klystronthermionic cathode operating at about $10 \mathrm{~A} / \mathrm{cm}^{2}$ is unity. Higher brightness can be achieved by operating such cathodes at higher current density; however, there is then a great degradation in the lifetime of the cathode. As with most proposed sources to power TeV linear colliders, the magnicon and gyrocon work best with high brightness beams. However, our initial estimates are that the magnicon will still work, although at reduced performance, with a conventional cathode.

Other possible cathodes under development by various groups include laser photocathodes and field emitters. Laser photocathodes operate by illuminating the cathode surface with a laser to stimulate photoemission and thereby achieve a high current density. Current densities up to $400 \mathrm{~A} / \mathrm{cm}^{2}$ have been achieved in this way. However, these are still experimental devices which must operate at very low pressure, typically below $10^{-11}$ torr to avoid cathode poisoning, and their lifetime is not well known. Field emitters are another potential type of cathode that is presently under development. NRL is active in research on field emitters, and this will be discussed in the following paragraph.

To start, we note that in many pulseline accelerator diodes there are very high fields at the cathode surfaces, so virtually any material surface will act as a field emitter. However, the field emission occurs when a surface plasma is generated by the high electric field at the surface. Only for a very short time (a few nanoseconds) is the cathode a true field emitter. At longer times, a surface plasma forms and the plasma is the electron source. This plasma expands at a typical rate of one to several centimeters per microsecond, so pulse length is severely limited as are the repetition rate, the average power, and the cathode lifetime. However, for single-shot experiments plasma field emitters have achieved very high beam brightness. Velvet and graphite cathodes can achieve brightness of 100 $\mathrm{kA} / \pi^{2} \mathrm{~cm}^{2} \mathrm{rad}^{2}$ fairly routinely.

Since the velvet or graphite emits easily, the strategy is to use these materials for emission surfaces and materials with good hold off and anodized aluminum or polished stainless steel for other surfaces. These other surfaces might be focusing electrodes for instance. Such a scheme has been shown to work at $30 \mathrm{~ns}$ pulse, but at high voltage. A significant issue is how well it will work for $1 \mu \mathrm{s}$ but at lower voltage. Even if the focusing electrodes hold off emission for $1 \mu \mathrm{s}$, the fact that a plasma forms means that the impedance will vary in time. A crucial question will be just how much of this microsecond of voltage pulse is usable with a plasma field-emitter cathode. Our proposed effort will devote some time to optimizing the beam pulse time.

Thus it is possible to do experimental work with high brightness beams, even though such cathodes do not extrapolate to the pulselengths and average power needed for driving $\mathrm{TeV}$ linear accelerators. Another advantage to plasma field-emitter cathodes is that these experiments could be performed relatively quickly; much more quickly than corresponding experiments on thermionic cathodes. This is so because, first, such cathodes have greatly reduced vacuum requirements $\left(10^{-4}\right.$ torr is usually sufficient ); and secondly, because there are fewer and expensive long lead time parts to acquire. Initial experiments could be done with high brightness beams generated by velvet or graphite cathodes. This would allow experiments to be performed while research on more appropriate cathodes proceeded separately.

The Naval Research Laboratory is now investigating the possibility of one type of field emitter cathodes. These cathodes are based on the manipulation of microtubules to engineer the microstructure of the cathode surface. These microtubules are biological structures derived from lipids [16]. They are typically tens of micrometers long one-half micrometer in diameter, and about a tenth of a micron in thickness and about $100 \AA$ radius of curvature at the tip. NRL has learned how to manipulate these tubules in many different ways. For instance, they can be coated with nickel, aligned with a magnetic field, and placed in an epoxy matrix. This can lead to an advanced cathode structure that would be a classic field-emitter; one that would not produce a cathode plasma. It has the potential of generating a high brightness beam from a cathode capable of high repetition rate and of high average power operation.

The cathode envisioned for a $\mathrm{TeV}$ supercollider might be described as a silicon lawn [17] (see Fig. 4). The cathode is composed of $10-\mu \mathrm{m}$ tall tubules protruding from an epoxy base, with an average tubule-tubule spacing of also $10-\mu \mathrm{m}$. The entire structure could be overcoated with a thin coating of gold, over silicon. The silicon is used for its characteristics of nonlinear current saturation, high thermal conductivity, and high melting point. Different levels of current saturation may be obtained by doping the 


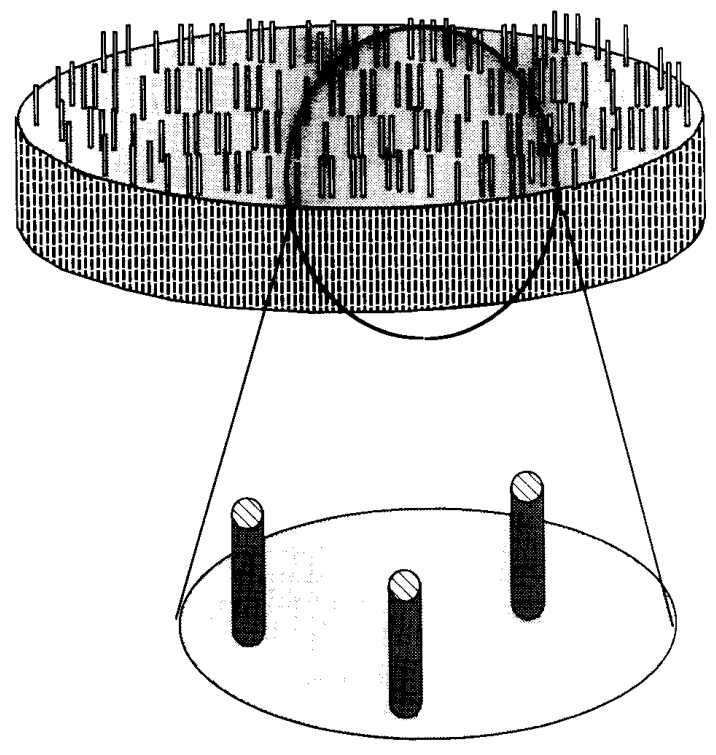

Fig. 4. The silicon "lawn" advanced cathode.

silicon overcoat [18]. Limiting the current to about 200$\mu \mathrm{A}$ per tip yields a current density of $200 \mathrm{~A} / \mathrm{cm}^{2}$. High brightness from such a cathode is anticipated in the light of its similarity to that of velvet.

\section{B. The Beam Acceleration and Transport System}

The beam is produced from a field-emitter cathode. In the initial experiments this would be velvet or graphite; in an actual system, an advanced cathode material would most likely be used, or as a fallback, a conventional thermionic emitter would be used. Following the emission of the electrons there are two stages: beam acceleration and beam focusing. To achieve a high quality beam, a staged electrostatic acceleration scheme would be used [19]. In [19], a 2-MeV 1-kA beam with a brightness of about 100 was produced routinely in this manner. The schematic of this acceleration scheme is shown in Fig. 5. An axial copper sulfate voltage divider is placed between the high voltage terminals of the accelerator. From this axial dividing shunt, electrodes can be attached at intermediate voltages determined by their positions on the shunt. In [19], an $80 \%$ transmitting molybdenum screen was placed in front of the cathode so that the beam would be uniform across the cathode face. In a high average power experiment, the diode would almost certainly have to be run without this screen, and the resulting beam might be less uniform than that in [19]. However, if the tubule cathode is successful, part of this inhomogeniety could be eliminated by adjusting the packing density.

The dynamics of the beam in the acceleration region is determined by the paraxial ray equation [20]:

$$
r_{b}^{\prime \prime}+\frac{\gamma^{\prime} r_{b}^{\prime}}{\beta^{2} \gamma}+\left[\frac{\gamma^{\prime \prime}}{2 \beta^{2} \gamma}+\frac{\Omega_{L}^{2}}{\beta^{2} c^{2}}\right] r_{b}-\frac{K}{r_{b}}-\frac{\epsilon^{2}}{r_{b}^{3}}=0
$$

where $r_{b}$ is the beam radius, $\gamma$ is the relativistic factor,

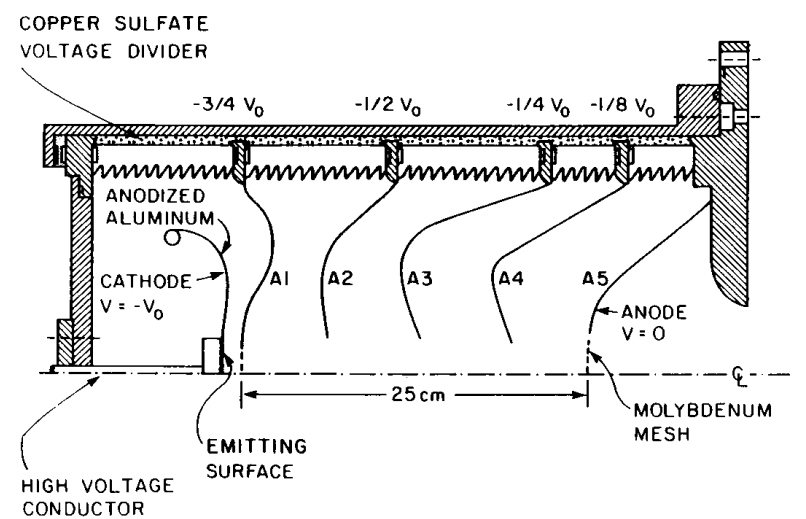

Fig. 5. Multi-Electrode Gun. The multistage $2-\mathrm{MeV} 1-\mathrm{kA}$ diode of reference [26].

related to the voltage $\mathrm{V}$ by $\gamma=1+\mathrm{eV} / m c^{2}, \beta=v_{z} / c$, $\Omega_{L}$ is the Brillouin frequency $e B / 2 m c$, where $B$ is the axial magnetic field and $K=2 e I / m \beta^{3} \gamma^{3} c^{3}$. We have assumed in (2) that the canonical momentum of the beam in the $\phi$ direction is zero. The parameter $K$ is determined from the outward electrostatic force of the beam, partially canceled by radial self-pinching. (That is, the outward force is reduced by a factor of $\gamma^{2}$.) If the voltage is that for one-dimensional relativistic space-charge-limited flow, then the paraxial ray equation gives the radius of the beam as a function of distance (assuming that $\Omega_{L}$ is zero in the acceleration region). The continuation of the equipotential surfaces from the edge of the beam can then be determined by the method of electrode synthesis [21][24]. This is the way in which the shape of the intermediate electrodes is determined in Fig. 5.

The beam exits the final electrode and proceeds towards the first cavity. In order that the beam does not spread out too much along its path, some sort of focusing system must be utilized. We consider here the simplest focusing, a thin magnetic lens. Taking the case of a $1-\mathrm{MeV} 100-\mathrm{A}$ beam, the paraxial ray equation becomes:

$$
r_{b}^{\prime \prime}+\left(\frac{\Omega_{L}}{\beta c}\right)^{2} r_{b}-\frac{5.2 \times 10^{-4}}{a}-\frac{1.1 \times 10^{-3}}{B a^{3}}=0
$$

in the region where there is no imposed electric field. Here $B$ is the beam brightness. We consider first a high brightness beam, $B=100$, and an initial radius, $r_{b 0}=1 \mathrm{~cm}$. This would correspond to a beam generated by a velvet or tubule field emitter at a current density of $30 \mathrm{~A} / \mathrm{cm}^{2}$. For the magnetic field we simplify to the case of a Gaussian profile,

$$
B(z)=B_{0} \exp -\left(\left(z-z_{0}\right) / \Delta\right)^{2}
$$

where $\Delta$ is taken to be $1.5 a_{0}$, and $a_{0}$ is the input radius of the beam. (An actual design would, of course, use realistic coils.) The solution for the high brightness beam is shown in Fig. 6 for the case of $B_{0}=460 \mathrm{G}$. Notice that the focus is about $40-\mathrm{cm}$ downstream, and that there is nearly a $20-\mathrm{cm}$ region where the beam radius is less than 


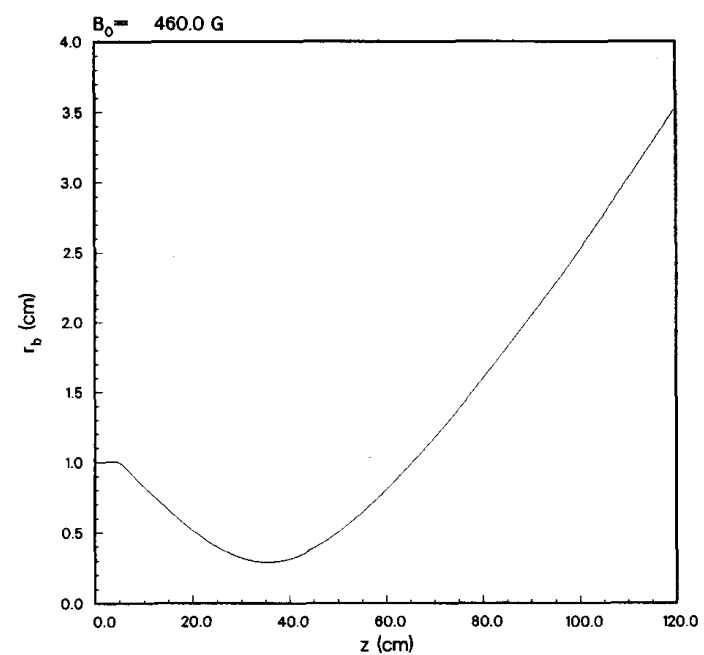

Fig. 6. Spatial dependence of the radius of a high brightness beam with a single magnetic focusing lens.

$3 \mathrm{~mm}$. Since a geometric spread in the beam radius means a spread in beam transverse energy at the second cavity, we consider the focus to be at the input to the second cavity. If a much sharper focus is required, then this is possible also, but at the price of a shorter focal region. Fig. 7 shows the computed beam envelop for a focusing field of $650 \mathrm{G}$. Notice that now there is a $5-\mathrm{cm}$ region where the beam radius is less than $1 \mathrm{~mm}$.

To conclude, we consider a low brightness beam. We consider here $b=1$, roughly what a SLAC klystron gun produces. We consider the initial beam radius to be $5 \mathrm{~cm}$. If the strength of the focusing field in $120 \mathrm{G}$, the beam envelope is shown in Fig. 8. The initial 5 -cm radius is of course too large to fit through the first cavity. However, $80 \mathrm{~cm}$ downstream the beam radius has been reduced to $1.5 \mathrm{~cm}$, most likely small enough to fit through the deflector cavity. About $30 \mathrm{~cm}$ further down stream, there is about a $7-\mathrm{cm}$ region where the beam focuses down to a radius below $0.5 \mathrm{~cm}$. Thus even with a low brightness beam, the magnicon should be a viable device, particularly as the focusing and transport system could almost certainly be optimized. However the power and efficiency will surely be degraded from that of a $B=100$ beam. The proposed NRL experiments will use high brightness beams generated by velvet or graphite cathodes in order to study the basic magnicon interaction. Our assumption is that high brightness cathodes of one sort or another will be available when it is time to manufacture the device. If this does not prove to be true, we feel that conventional cathodes could also be used with some penalty in power and efficiency. Also, lower voltages could be used, but again, at reduced performance.

We now consider the input of the deflected beam through the half cusp at the entrance to the extraction cavity. The conservation of canonical momentum in the theta direction is the crucial consideration here. Since the electron is born on the diode with no magnetic field and no

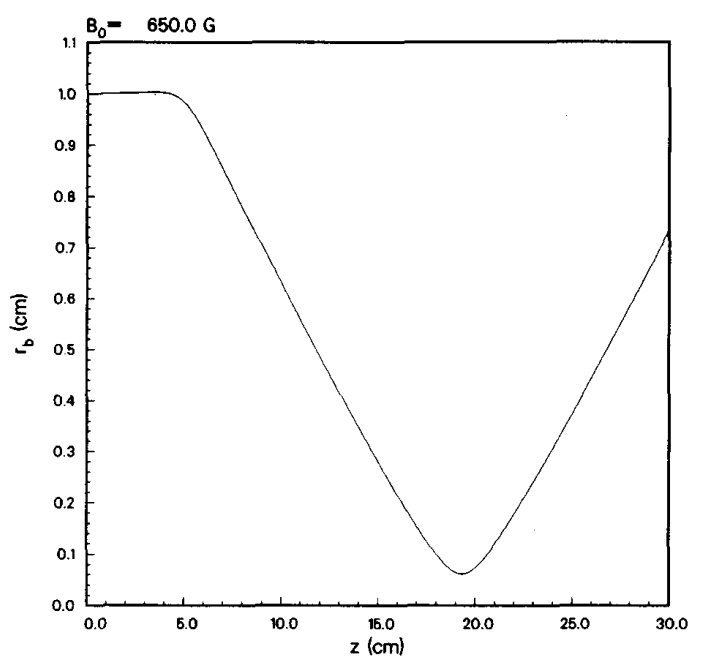

Fig. 7. Spatial dependence of a high brightness beam with stronger focusing.

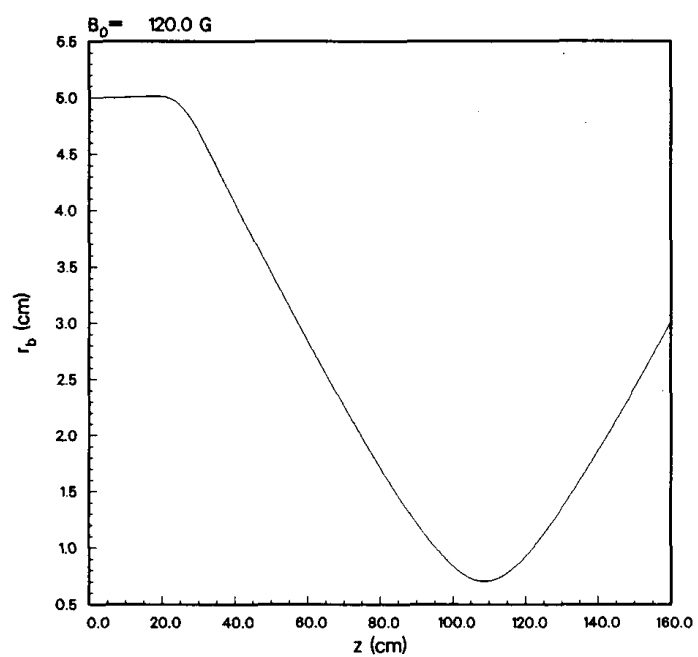

Fig. 8. Spatial dependence of the radius of a low brightness beam.

theta velocity

$$
P_{\theta}=-e r A_{\theta} / c+\gamma m v_{\theta} r=0
$$

where $A_{\theta}=B r^{2} / 2$. If the entrance to the second cavity is an ideal half cusp, then inside the cavity,

$$
v_{\theta}=e B r / 2 m \gamma c, \quad v_{r}=0 .
$$

Thus the Larmor radius is one-half the entrance radius; the particle passes through the axis at the point on its Larmor orbit opposite to where it entered the cusp. Clearly, any spread in input radius will translate into a spread in perpendicular velocity in the second cavity. Thus it is important to keep the input radius spread as small as possible; this is clearly one of the main advantages to high brightness beams. 
Since the cavity is resonant at $X$-band, to obtain cyclotron resonance the magnetic field in the cavity is about 10 $\mathrm{kG}$ for a $1-\mathrm{MeV}$ beam. Thus the entrance point of the cavity should be at a deflection of about $1 \mathrm{~cm}$ if the beam enters a perfect half cusp at the full field. Since generating a perfect half cusp is difficult at a field of $10 \mathrm{kG}$, we have examined the orbits of particles as they enter an open solenoid with a coil radius of $5 \mathrm{~cm}$ and a center field of 10 kG. In Fig. 9(a) is shown the orbit radius and the alpha value ( $\alpha=v_{1} / v_{z}$ ) as a function of $z$ for an entrance radius of $1.5 \mathrm{~cm}$. In Fig. 9(b) is shown the same thing for an input radius of $1.75 \mathrm{~cm}$. Clearly, this spread of input radius with either an ideal cusp or a gradual field transition leads to a large spread in alpha. With a high brightness beam, such a spread could still give rise to acceptable performance. However, a low brightness beam would probably not work with such an input scheme. It is likely that an important intermediate step in an experiment is to measure beam quality on entering the cusp, but in the absence of an RF-drive of the first cavity. A possible means to do so is to propagate the beam into an offset cusp and to measure the resulting spread in beam alpha. The entrance to the half solenoid is actually the worst possible scenario. The field profile could be sharpened up either by varying the current profile along the solenoid, or by using magnetic materials. Fortunately, codes are now commercially available which are design tools for magnetic field systems in nearly any three dimensional configuration.

Another option for the magnetic circuit is a half cusp at low field followed by field compression. Let us say that in the first deflector cavity the beam is deflected by an angle $\beta$. Then the vertical displacement of the beam after a horizontal distance $L_{0}$ is given by $L_{0} \tan \beta$, and the transverse velocity is given by

$$
v_{t}=L_{0} \Omega_{L 0} \tan \beta / \gamma
$$

at the entrance of a perfect cusp. Here, $\Omega_{L 0}$ is the Brillouin frequency at the entrance. Upon entrance to the cusp, the magnetic field is compressed to the final $10-\mathrm{kG}$ value (characterized by $\Omega$ ). Assuming magnetic compression, the transverse velocity at the final field is given by

$$
v_{t}=\left(\Omega_{0} \Omega\right)^{1 / 2} L_{0} \tan \beta / \gamma .
$$

Thus the cusp can be at smaller field than $10 \mathrm{kG}$, but the propagation distance to the cusp entrance must be longer in order that the transverse velocity be the same.

To continue, we consider the possibility of a current limit in the output cavity, since the beam has a large alpha and is near the axis. The formula for limiting current is [25]

$$
I_{L}(K A)=\frac{8.5}{\ln \left(\frac{a}{r_{b}}\right)}\left[\gamma^{2 / 3}-\left(\frac{1+\gamma^{2} \alpha^{2}}{1+\alpha^{2}}\right)^{1 / 3}\right]^{3 / 2} .
$$

The beam radius $r_{b}$ is about $c / \omega$, while the wall radius is $3.8 \mathrm{c} / \omega$. If alpha is 2 , then the limiting current is about

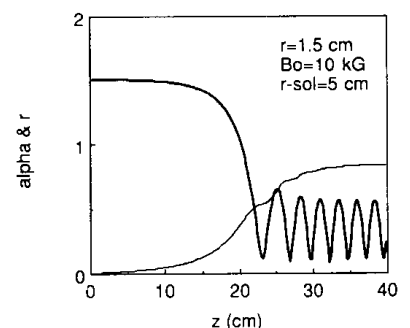

(a)

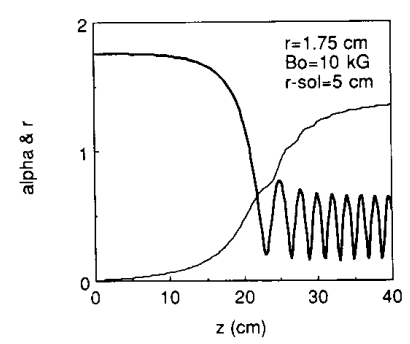

(b)

Fig. 9. Spatial dependence of beam alpha $(\alpha)$ (the monotonic curve) and beam radius (the oscillating curve) entering a solenoid (a) at a radius of $1.5 \mathrm{~cm}$, and (b) at an entrance radius of $1.75 \mathrm{~cm}$.

$300 \mathrm{~A}$. Thus the 100 - A beam we propose is well below the limiting current. Furthermore, at the input to the cavity the beam is near the grounded end wall, and as it propagates into the center of the cavity it loses perpendicular energy. Thus the electrostatic current limit is in all likelihood much greater than the $300 \mathrm{~A}$ given by (9). We do not expect the electrostatic current limit to be an obstacle to achieving magnicon performance.

To conclude, our initial studies give us confidence that there are many possible options for the beam transport system which will allow for a design of a high-power highefficiency $X$-band magnicon. The task is much easier with a high brightness beam, but it is still possible, at reduced performance, with a lower brightness beam. We have no doubt that the beam transport for an $X$-band magnicon using the new NRL accelerator and a graphite or velvet cathode can be accomplished.

\section{Beam Deflection in the Input Cavity}

As in the Soviet experiments, we consider both the input and output cavity to be operating in a $\mathrm{TM}_{11}$ mode. This mode is at cutoff, so the fields are given by

$$
\begin{aligned}
& E_{z}=\operatorname{Re} h E_{0} J_{1}\left(k_{t} r\right) \exp i(\phi-\omega t) \\
& B_{r}=(c / \omega r) E_{z} \\
& B_{\theta}=\operatorname{Re} i h J_{1}^{\prime}\left(k_{t} r\right) h E_{0} \exp i(\phi-\omega t)
\end{aligned}
$$

where $h$ is chosen so that the field is normalized as

$$
\int d^{3} r E_{z}^{*} E_{z}=k_{t}^{-2} L E_{0}^{2}
$$


where $L$ is the cavity length. Of course, for a cutoff mode, $\omega=c k_{t}$. The quantity $a$ is then given by

$$
h=\left\{\pi^{0.5} k_{t} a J_{1}\left(k_{t} a\right)\right\}^{-1}
$$

where $a$ is the radius of the cavity wall. For the lowest order radial $\mathrm{TM}_{110}$ mode, we find that $h=0.21$ and $k_{t} a$ $=3.8$. Near the center of the cavity, where the beam propagates, we find that

$$
\begin{aligned}
& B_{x}=0.11 E_{0} \cos \omega t \\
& B_{y}=0.11 E_{0} \sin \omega t \\
& E_{z}=0.11 E_{0} k_{t}(x \cos \omega t+y \sin \omega t)
\end{aligned}
$$

where we have used real notation here. Where convenient, we will shift back and forth between real and complex notation.

As the electron enters the deflection cavity, it is deflected by rotating magnetic field in the cavity. The equations of motion in the first cavity are

$$
\begin{aligned}
\gamma m \frac{d v_{x}}{d t} & =\frac{e v_{t} h}{2 c} E_{0} \sin \omega t \\
\gamma m \frac{d v_{y}}{d t} & =-\frac{e v_{-} h}{2 c} E_{0} \cos \omega t .
\end{aligned}
$$

If the electron enters the cavity at time $t_{0}$, the velocity is given by

$$
\begin{aligned}
& v_{x}=-\frac{e v_{z} h E_{0}}{2 \gamma m c \omega}\left[\cos \omega t-\cos \omega t_{0}\right] \\
& v_{y}=-\frac{e v_{z} h E_{0}}{2 \gamma m c \omega}\left[\sin \omega t-\sin \omega t_{0}\right] .
\end{aligned}
$$

Thus the polar angle $\alpha$ at the exit of the deflection cavity, i.e., $t=t_{0}+L / V_{*}$, is given by

$$
\alpha=\frac{\left(v_{x}^{2}+v_{y}^{2}\right)^{1 / 2}}{v_{z}}=\frac{e h E_{0}}{\gamma m c \omega}\left[1-\cos \frac{\omega L}{v_{z}}\right] .
$$

Since the cavity will be designed for maximum deflection angle, that is, maximum alpha, the length will be set by $\omega L / v_{z}=\pi$. The azimuthal angle $\phi$ at the exit of the deflection cavity is given by

$$
\phi=\frac{\omega L}{2 v_{z}}+\omega t_{0} .
$$

Integrating the equations of motion one more time, we find:

$$
\begin{aligned}
\left(\begin{array}{l}
x \\
y
\end{array}\right)= & \frac{-e v_{-} h E_{0}}{2 m \gamma c \omega}\left[\frac{e^{-i\left(\left(\omega_{i} / v_{i}\right)+\omega_{i}\right)}-e^{\left.-i \omega t_{0}\right)}}{-i \omega}\right. \\
& -\frac{z}{v_{z}} e^{\left.-i \omega t_{i}\right)} \mid\left(\begin{array}{l}
1 \\
i
\end{array}\right)
\end{aligned}
$$

where we have gone back to complex notation for the displacement. This will be more convenient in the next section.

\section{Design of the Input Cavity}

The first issue in the design of the input cavity is the design of the input mode launcher. This input launcher will be designed to launch a rotating $\mathrm{TM}_{11}$ mode. As was done in [3], this will be done with two sidewall slots phased $90^{\circ}$ apart. The size of the holes will depend on the $Q$ of the cavity. Both the beam loading and the wall resistivity contribute to the $Q$. At critical coupling, the input and output $Q$ 's are equal, the field in the cavity maximizes and the reflected power is zero. The other concern is the resonant frequency of the input cavity. The beam loading has an important effect on the resonant frequency and this must be accounted for in an actual design.

To continue, we consider the beam loading of the input cavity, which affects both the $Q$ and resonant frequency. We calculate this by using a Vlasov-Maxwell formulation. The electric field in the cavity is determined by

$$
\nabla^{2} E_{z}-\frac{1}{c^{2}} \frac{\partial^{2} E_{z}}{\partial t^{2}}=\frac{4 \pi}{c^{2}} \frac{\partial J_{z}}{\partial t} .
$$

We have neglected space charge, which is exactly true if there is no $z$ variation, as is the case for a $\mathrm{TM}_{110}$ mode, and is approximately true otherwise. We assume that the electric field is written as the sum of orthogonal basis functions:

$$
E_{:}=\sum_{n} E_{n} \chi_{n}\left(\vec{r}_{\perp}, z\right)
$$

The functions are eigenfunctions of the left side of (19) and are normalized according to (11). The basis function for the $\mathrm{TM}_{110}$ mode is given by (10). Multiplying (19) by $\chi_{i}^{*}$ (the basic function for the $\mathrm{TM}_{110}$ mode) and integrating over the volume of the cavity and time from zero to $2 \pi / \omega$, we find

$$
\begin{aligned}
& \frac{2 \pi}{\omega} k_{t}^{-2} L\left(\frac{\omega^{2}}{c^{2}}-k_{t}^{2}\right) E_{0} \\
& \quad=\frac{1}{\sqrt{\pi} k_{t} a J_{1}^{\prime}\left(k_{t} a\right)}\left(\frac{-4 \pi i \omega}{c^{2}}\right) \int d^{3} r \int_{0}^{2(\pi / \omega)} \\
& \cdot d t J_{z}(r, \omega) J_{1}\left(k_{t} r\right) e^{-i(\theta-i \omega t)} .
\end{aligned}
$$

The current in the $z$ direction is given by

$$
\mu_{z}=-e \int f(\vec{r}, \vec{p}, t) v_{z} d^{3} \vec{p}
$$

where the distribution function $f$ is given by

$$
\begin{aligned}
f= & \frac{I}{e} \int_{\infty}^{\infty} d t_{0} \delta\left(z-\xi\left(t_{0}, t\right)\right) \delta\left(\vec{r}_{\perp}-\vec{r}_{\perp}\left(t_{0}, t\right)\right) \\
& \cdot \delta\left(\vec{p}-\vec{\eta}\left(t_{0}, t\right)\right) .
\end{aligned}
$$

Here $I$ is the beam current and $\xi\left(t_{0}, t\right)$ is the axial position of a particle at time $t$ if it entered at time $t_{0}$. Analogous definitions apply for $\vec{r}_{\perp}\left(t_{0}, t\right)$ and $\vec{\eta}\left(t_{0}, t\right)$.

The particle is characterized only by its entrance time $t_{0}$. The integral over $t_{0}$ is a sum over all particles. Using the fact that for small arguments, $J_{1}\left(k_{t} r\right) e^{-i \theta}=x-i y$, 
we find that Maxwell's equation can be reduced to:

$$
\begin{aligned}
\left(\frac{\omega^{2}}{c^{2}}-k_{t}^{2}\right) E_{0}= & \frac{\omega k_{t}^{2} I}{4 \pi^{3 / 2} a L J_{1}^{\prime}\left(k_{t} a\right)}\left(\frac{4 \pi i \omega}{c^{2}}\right) \int_{0}^{L} d z \int_{0}^{2 \pi / \omega} \\
& \cdot d t_{0}\left[x\left(t_{0}, t\right)-i y\left(t_{0}, t\right)\right] \exp i \omega(t) .
\end{aligned}
$$

In the exponential above, $t$ denotes the time of a particle at position $z$ which entered at $t_{0}$. In obtaining (24), we have reversed the $t$ and $t_{0}$ integrals [26], [27]. To calculate the loading in the linear regime, use the linear values for $x$ and $y$ and use $t=t_{0}+z / v_{3}$. Using the complex expressions for the particle deflection from (18), we find that the dispersion relation of the input cavity is given by

$$
\begin{aligned}
\left(\frac{\omega^{2}}{c^{2}}-k_{t}^{2}\right)= & \frac{4 k_{t} e I v_{z}^{2}}{2 a^{2} L\left[J_{1}^{\prime}\left(k_{t} a\right)\right]^{2} m \gamma c^{3} \omega^{2}} \mid \frac{L \omega}{v_{z}}-2 \sin \frac{L \omega}{v_{z}} \\
& \cdot \cos \frac{L \omega}{v_{z}}+i\left\{\left(2 \cos \frac{L \omega}{v_{z}}-2\right)\right. \\
& \left.+\frac{L \omega}{v_{z}} \sin \frac{L \omega}{v_{z}}\right\} \mid
\end{aligned}
$$

Notice that on the right-hand side there is a real part corresponding to a frequency shift, and an imaginary part, which for $\omega L / v_{z}=\pi$ corresponds to a damping. These two are of about the same order of magnitude. Finally, we point out that the $\mathrm{TM}_{110}$ mode is actually degenerate with the $\mathrm{TM}_{110}$ mode of opposite rotation. However, the input coupler selects one rotation. It is not difficult to see, from the preceding analysis, that the beam does not couple the two rotation directions to one another. The $\mathrm{TM}_{110}$ mode should not be degenerate with the $\mathrm{TE}_{01}$ mode, because the $k_{z}$ 's for the two are quite different and the $k_{z}$ is comparable to $k_{t}$.

Utilizing the dispersion relation to get the beam-loaded damping and frequency shift, we can then come up with the cavity design. For a frequency of $10 \mathrm{GHz}, L=1.5$ $\mathrm{cm}$ and $a=2 \mathrm{~cm}$ if $v_{t} / v_{z}=2$. For now we will not consider the effect of the beam-loaded frequency shift, although it must be considered in an actual cavity design. The damping rate is related to $Q$ by $\omega / 2 Q$. From the expression for damping rate for the $10-\mathrm{GHz}$ frequency for the $1-\mathrm{MeV} 100-\mathrm{A}$ beam, we find that the beam-loaded $Q$, $Q_{b}$ is given by $Q_{b}^{-1}=6 \times 10^{-4}$. The ohmic $Q, Q_{0}$, for a $\mathrm{TM}_{110}$ mode is given by

$$
Q_{O}^{-1}=\omega(1+a / L) \delta_{s} / 3.8 c
$$

where $\delta_{s}$ is the skin depth. Taking a skin depth of $6.6 \times$ $10^{-5} \mathrm{~cm}$ for copper at $X$-band, we find $Q_{o}^{-1}=0.9 \times 10^{-4}$. This $Q^{-1}$ is smaller than that of the beam. Furthermore, such a high $Q$ does not allow the accelerator to respond sufficiently quickly to changes in drive. We assume the total cavity $Q$ (including the beam loading) can be lowered to about 400 by azimuthal stots [28] or else by using a more resistive material in the walls. At a $Q$ of 400 , the beam loading is a small perturbation to the total $Q$ rather than the dominant factor. Thus the total reciprocal $Q$ is about $2.5 \times 10^{-3}$. The power input to the cavity is then:

$$
P=\omega E_{0}^{2} L / 8 \pi Q k_{1}^{2} \text {. }
$$

Using the parameters we have determined, we find

$$
P(\mathrm{~W})=2 \times 10^{-1} E_{0}^{2} \text {. }
$$

Thus with $600 \mathrm{~kW}$ of input power, the electric field is about 1600 in metric units and the deflection angle $(\alpha)$ is about $7 \times 10^{-2}$ rad. With a $1-\mathrm{MW}$ driver, which at $X$-band is a commercial item available at reasonable price, it should not be difficult to drive the first cavity. Thus the first cut at the design of the NRL magnicon will use a single deflection cavity, unlike the Soviet design. NRL will rely on the use of a high power driver and an amplification of less than $20 \mathrm{~dB}$. With a lower power driver, a high power magnicon is still a possible concept, but additional deflection cavities would be required as discussed by Karliner et al. [1]. Our choice is to utilize the highest power driver available, as long as it has the required frequency stability, so as to eliminate additional deflection cavities and thereby simplify as much as possible the initial experiment. In follow on experiment designed for maximum gain, additional cavities will be utilized.

\section{E. Orbits in the Output Cavity}

To find the energy loss in the output cavity, we begin by considering first the orbits of the particles there. In the next section, we continue by actually calculating the beam loading as was done for the input cavity. In calculating the particle orbits it is most convenient often to use as dependent variables quantities which are constant in the presence of the magnetic field but in the absence of the radiation field. These are the perpendicular and parallel components of particle momentum, and the $x$ and $y$ components of the guiding center coordinate of the particle. Assuming that the fields near the axis are as given in (13), then the equations of motion are

$$
\begin{aligned}
\frac{d}{d t} p_{z} & =-\frac{e E_{0} h k_{t}}{2}(x \cos \omega t+y \sin \omega t) \\
\frac{d}{d t} p_{x}+m \Omega v_{y} & =\frac{e}{c} v_{-} \frac{h}{2} E_{0} \sin \omega t \\
\frac{d}{d t} p_{y}-m \Omega v_{x} & =-\frac{e}{c} v_{-} \frac{h}{2} E_{0} \cos \omega t .
\end{aligned}
$$

Transforming the new variables

$$
\begin{array}{rlrl}
p_{x} & =p_{t} \cos \phi, & p_{y} & =p_{1} \sin \phi \\
x-\frac{p_{y}}{m \Omega} & =x_{G}, & y & y+\frac{p_{x}}{m \Omega}=y_{G}
\end{array}
$$

the equations of motion become

$$
\begin{aligned}
v_{*} \frac{d}{d z} \frac{p_{\Sigma}^{2}}{2}= & -\frac{p_{\mathcal{z}} c E_{0} k_{t} h}{2}\left(x_{G} \cos \omega t+y_{G} \sin \omega t\right) \\
& -\frac{e E_{0} h p_{t} p_{z}}{2 m} \sin (\phi-\omega t)\left|\frac{k_{t}}{\Omega}-\frac{1}{\gamma c}\right|
\end{aligned}
$$




$$
\begin{aligned}
\frac{1}{2} v_{*} \frac{d}{d z} p_{t}^{2} & =-\frac{e}{c} \frac{p_{z} p_{t}}{m \gamma} \frac{h}{2} E_{0} \sin (\phi-\omega t) \\
v_{z} \frac{d \phi}{d z} & =\frac{\Omega}{\gamma}+\frac{e p_{z} h}{m \gamma p_{t}} E_{0} \cos (\phi-\omega t) \\
\frac{d t}{d z} & =\frac{1}{v_{z}}=\frac{\gamma m}{p_{*}} \\
v_{z} \frac{d x_{\mathrm{G}}}{d z} & =\frac{e v_{z} h E_{0}}{2 m \Omega c} \cos \omega t \\
v_{z} \frac{d y_{G}}{d z} & =\frac{e v_{z} h E_{0}}{2 m \Omega c} \sin \omega t .
\end{aligned}
$$

Here we have used $z$ instead of $t$ as the independent variable. The equations are then supplemented by (31d), which expresses $t$ in terms of $z$. Notice that the equation for $p_{z}^{2}$ has one term proportional to $\sin (\phi-\omega t)$ and other terms proportional to, for instance, $\cos \omega t$. Since $\phi$ is nearly equal to $\omega t$ near cyclotron resonance, the former term varies very slowly, whereas the latter oscillates rapidly about a zero mean. Thus the change in $p_{z}$, averaged over many cyclotron periods, is dominated by the first term. Considering only such terms in (31), we look at is the change of $p_{z}$ with respect to $p_{t}$. Combining (31a) and (31b), we find:

$$
\frac{d p_{l}^{2}}{d p^{2}}=\frac{1}{\frac{\gamma k_{\perp} c}{\Omega}-1}
$$

If the frequency is at cyclotron resonance at the input of the extraction cavity, then there is no change in $p_{z}$. If the frequency is near cyclotron resonance, the change in parallel momentum is much less than the change in perpendicular momentum. Thus even though the electric field is only in the $z$ direction, the particle momentum loss is only in the transverse direction. Clearly, the magnetic field takes energy out of the perpendicular direction and puts it into the parallel direction. However, the electric field just takes away the parallel energy put there by the magnetic field. Thus the electric field takes energy out of the parallel motion, and the magnetic field transforms this into a loss of transverse energy. Clearly, the only energy available to drive the magnicon then is the transverse energy of the beam. However, if the transverse efficiency can be near $100 \%$, the spent beam will be nearly monoenergetic and have nearly all of its energy in the $z$ direction. A large fraction of the spent beam energy could almost certainly be regained with a depressed collector.

Making the same approximation, we find that an equation for the gamma of the particle is

$$
\frac{d \gamma}{d z}=\frac{-k_{t} e E_{0} p_{t} h}{2 \Omega m^{2} c^{2}} \sin (\phi-\omega t) \text {. }
$$

The actual design of the output resonator will be done with an orbit integrator, as is done in the case of gyrotron design. As a first approximation, we consider the unperturbed orbit of the particle. Notice, however, that for the magnicon, unlike the gyrotron, the initial cyclotron phase of all particles is the same:

$$
\phi_{0}=\rho_{0}+\omega t_{0}, \quad \phi=\phi_{0}+\frac{\Omega}{\gamma} \frac{z}{v_{z}}
$$

where $\rho_{0}$ is an initial phase which is the same for all particles. For the case of $\rho_{0}=\pi / 2$ (we will see in the next section that $\rho_{0}$ is controlled by the frequency of the driver), and $\Omega / \gamma=\omega$, and using the fact that $t=t_{0}+z / v_{-}$, we find:

$$
\frac{d \gamma}{d z}=-\frac{k_{t} e E h p_{t}}{2 m^{2} c^{2} \Omega} \equiv \epsilon .
$$

For an $\alpha=2$ beam the maximum efficiency is $80 \%$, so we adjust the electric field and cavity length so that $\gamma_{0}-$ $\gamma(L)=0.8\left(\gamma_{0}-1\right)$ and find

$$
\epsilon L=0.8\left(\gamma_{0}-1\right)
$$

for the theoretical efficiency. This specifies the $E L$ product in the output cavity. Let us assume that the end wall of the cavity can tolerate a maximum field. The radial position is that where the Bessel function maximizes. This position is about halfway to the sidewall and, $J_{1}$ maximizes at a value of 0.58 . Then considering that $h=0.22$, a maximum field strength of $2 \times 10^{5} \mathrm{~V} / \mathrm{cm}$ implies $E_{0}=$ $5.6 \times 10^{3}$ in metric units. Hence we find that the length of the output cavity $L$ is about $6 \mathrm{~cm}$. Now let us examine what a thermal spread on the beam will do to the efficiency. We first consider the effect of a spread of $\rho_{0}$ about $\pi / 2$. Assuming a Gaussian distribution of $\rho_{0}$ about $\pi / 2$, we find that the average change in $\gamma$ after the distance $L$ is given by

$$
\begin{aligned}
\Delta \gamma & =\frac{\epsilon L}{\sqrt{\pi} \Delta \rho} \int_{\infty}^{\infty} \rho \exp -\frac{\left(\rho_{0}-\frac{\pi}{2}\right)^{2}}{(\Delta \rho)^{2}} \sin \rho_{0} \\
& =\epsilon L \exp -\frac{(\Delta \rho)^{2}}{4}
\end{aligned}
$$

where $\Delta \rho$ is assumed small. (It is clear that if $\rho_{0}$ were distributed uniformly from zero to $2 \pi$, the energy loss would be zero.) Thus the efficiency is relatively insensitive to spread in $\rho_{0}$; a spread of 1 rad only reduces the transverse efficiency by $20 \%$. Now let us examine a spread in polar angle, which as we have seen in the previous section, can be induced by a spread on input radii at the entrance to the cusp field. At $\rho_{0}=\pi / 2$, the expression for energy change is

$$
\Delta \gamma=-\int \frac{L k_{t} e E h p \sin \alpha}{2 m^{2} c^{2} \Omega} f(\alpha) d \alpha
$$

where

$$
\int f(\alpha) d \alpha=1
$$

Let us take for $f(\alpha)$ a waterbag distribution having value between $(\Delta \alpha)^{-1}$ between $\alpha_{0}-\Delta \alpha / 2$ and $\alpha_{0}+\Delta \alpha / 2$. 
We assume that the efficiency maximizes where the particle with the smallest value of transverse momentum loses all perpendicular momentum. Then the ratio of energy loss of the thermal distribution of $\alpha$ to that of the cold distribution is $\sin \left(\alpha_{0}-\Delta \alpha / z\right) / \sin \alpha_{0}$. Again the degradation of efficiency is not large.

\section{F. Design of the Output Cavity}

Here we determine the design of the output cavity and also the criterion for frequency stability of the driver assuming the unperturbed orbit in the output cavity. The actual design will use the numerically computed orbits as in the case of gyrotron design. The cavity design is determined by the Vlasov equation, as in Section III-D:

$$
\begin{aligned}
& {\left[\frac{\omega\left(\omega+\frac{i k_{t} c}{Q}\right)}{c^{2}}-k_{t}^{2}\right] E_{0}} \\
& =i A \int_{0}^{L} d z \int_{0}^{2 \pi} d t_{0}\left[x\left(t_{0}, t_{0}+\frac{z}{v_{z}}\right)\right. \\
& \left.\quad-i y\left(t_{0}, t_{0}+\frac{z}{v_{z}}\right)\right] \exp i\left(\omega t_{0}+\frac{\omega z}{v_{z}}\right)
\end{aligned}
$$

where

$$
A=\frac{\omega}{2 \pi^{3 / 2}} \frac{k_{l}^{2} I}{2 a J_{1}^{\prime}\left(k_{l} a\right) L}\left(\frac{4 \pi \omega}{c^{2}}\right)
$$

and $Q$ on the left-hand side denotes the output coupling and ohmic heating of the cavity wall. Putting in the unperturbed orbit,

$$
\begin{aligned}
x= & \frac{p_{t}}{m \Omega}\left\{\exp -i\left(\rho_{0}-\pi / 2\right)\right. \\
& \left.\quad-\exp -i\left(\rho_{0}-\pi / 2+\frac{\Omega}{\gamma} \frac{z}{v_{z}}\right)\right\} \\
y= & i x
\end{aligned}
$$

we can take the real and imaginary parts of the nonlinear dispersion relation and get the result:

$$
\begin{aligned}
\frac{\omega k_{t}}{Q c} E_{0}= & 2 A \frac{p_{t}}{m \Omega} \frac{2 \pi}{\omega^{2}} v_{z}\left[-\cos \left(\frac{\omega L}{v_{z}}-\rho_{0}\right)\right. \\
& \left.+\cos \rho_{0}+\frac{L \omega}{v_{z}} \sin \rho_{0}\right] \\
\left.\frac{\omega^{2}}{c^{2}}-k_{t}^{2}\right\rfloor E= & 2 A \frac{p_{t}}{m \Omega} \frac{2 \pi}{\omega^{2}} v_{z}\left[\sin \left(\frac{\omega L}{v_{z}}-\rho_{0}\right)\right. \\
& \left.-\sin \rho_{0}-\frac{L \omega}{v_{z}} \cos \rho_{0}\right] .
\end{aligned}
$$

Since $L \omega / v_{-}$is about 20 for the beam and cavity we are considering, the last term in the brackets on the right-hand sides of (40a) and (40b) are dominant.

The imaginary part (40a) determines the $Q$ of the cavity. The output power of the cavity maximizes $\rho_{0}=\pi / 2$ as determined in the previous section. Putting in numbers for the beam we have specified, we find that the $Q$ of the cavity is about 150. A small part of the reciprocal $Q$ is the ohmic $Q$. The remainder is the output $Q$. As in the Soviet experiment, the output will be taken from a radial coupling hole in the sidewall of the cavity.

Up to now, we have simply assumed that the value of the input phase is $\pi / 2$ to maximize the power extraction. The question is how to obtain this value for the phase. As is apparent from (40b), the phase is determined from the frequency shift. For the beam that we have considered, we find that the frequency shift is about $1 \%$ from the empty cavity frequency.

As the phase varies from zero to $2 \pi$, the frequency shift varies over about this range. However, only for the value of frequency shift corresponding to $\rho_{0}=\pi / 2$ is the power output maximized. The output depends sensitively on the frequency of the driver then. For optimum performance of the magnicon, the driver frequency must be stable to less than $1 \%$. However, the reciprocal $Q$ of the input cavity gives a smaller operating window in frequency. Thus the frequency of the input cavity must be carefully designed, and the design must include the beam loading of the output cavity. This then completes the conceptual design of the $10-\mathrm{GHz}$, single deflector cavity magnicon. A schematic of the NRL magnicon is shown in Fig. 10.

\section{G. Operation at the Second Harmonic}

While $X$-band is a possible operating frequency for the $\mathrm{TeV}$ linear supercollider, the preferred frequency is about $17 \mathrm{GHz}$ [6], [7]. To achieve such a frequency, one option would be harmonic operation; that is, deflect the beam at $10 \mathrm{GHz}$ where a powerful driver is available, but design the output cavity to run at $20 \mathrm{GHz}$, the second harmonic of the cyclotron frequency there. To operate at the harmonic, one would use a $\mathrm{TM}_{22}$ mode in the output cavity. The field there is given by

$$
E_{z}=h E_{0} J_{2}\left(k_{t} r\right) \exp i(2 \phi-\omega t)
$$

where the normalization is as for the $\mathrm{TM}_{11}$ mode. Thus

$$
h=\left\{\sqrt{\pi} J_{2}^{\prime}\left(k_{t} a\right) k_{t} a\right\}^{-1}
$$

and $k_{1} a=5.1$ and the maximum value of the Bessel function is 0.49 at $k_{1} r=3$. To calculate the energy loss of the beam, use the unperturbed orbits:

$$
\begin{aligned}
& x=\frac{p_{t}}{m \Omega}\left(\sin \phi_{0}+\sin \left(\phi_{0}+\frac{\Omega}{\gamma}\left(t-t_{0}\right)\right)\right) \\
& y=\frac{-p_{t}}{m \Omega}\left(\cos \phi_{0}+\cos \left(\phi_{0}+\frac{\Omega}{\gamma}\left(t-t_{0}\right)\right)\right)
\end{aligned}
$$

where we have used real notation, and the equation for the rate of change of the relativistic $\gamma$ with $z$

$$
d \gamma / d z=-\left(e E_{z}(z) / m c^{2}\right) .
$$

Near the axis of the cavity, the $z$ component of the electric field is given by 


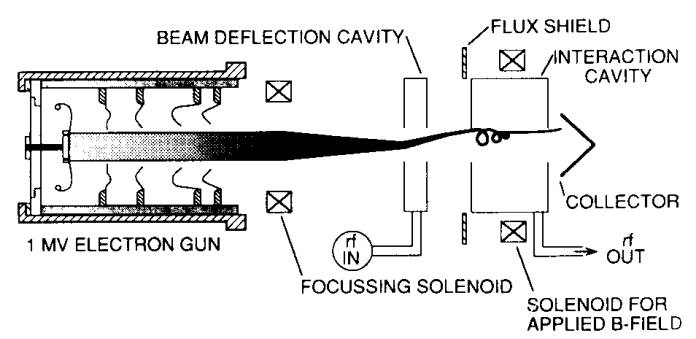

Fig. 10. Schematic of the proposed NRL magnicon experiment.

$$
E_{z}=-\frac{h E_{0} k_{t}^{2}}{8}\left\{\left(x^{2}-y^{2}\right) \cos \omega t+2 x y \sin \omega t\right\} .
$$

Inserting the unperturbed orbit in (46), assuming $\phi_{0}=\rho_{0}$ $+\omega t_{0} / 2 \omega=2 \Omega / \gamma$, we find that

$$
\frac{d \gamma}{d z}=-\frac{e h E_{0} k_{t}^{2}}{8 m c^{2}}\left(\frac{\gamma v_{t}}{\Omega}\right) \sin 2 \rho_{0}
$$

where we have neglected the contributions to $d \gamma / d z$ which oscillate rapidly in $z$. Taking $\rho_{0}=\pi / 4$, we calculate the energy loss as in Section III-E. For an $\alpha=2$ beam, the condition for $100 \%$ transverse efficiency is

$$
\frac{e h E_{0} k_{t}^{2} \gamma v_{t}}{8 m c^{2} \Omega} L=1.6
$$

Assuming as before that the cavity end wall can withstand an RF field of $200 \mathrm{kV} / \mathrm{cm}$, we find that the cavity length is again about $6 \mathrm{~cm}$. Since the cavity dimensions and beam parameters are about the same as in the $X$-band case, the cavity $Q$ will also be about the same. Thus as a followon experiment, we feel that a harmonic magnicon can be designed and built. This would then be a potential source for the powering of a $\mathrm{TeV}$ linear supercollider at a frequency of $17 \mathrm{GHz}$. The efficiency would be between about 50 and $80 \%$ and the power would be in the range of 50 $80 \mathrm{MW}$.

\section{H. Operation at High Amplification}

So far, we have addressed the issue of power, coherence, and harmonic operation in the proposed NRL magnicon development project. For accelerator applications, the total gain is also an important parameter. If the gain of each tube is high enough, one could envision the entire accelerator controlled by a single master oscillator. As we have seen, the initial NRL experiment is designed only for a gain of about $17 \mathrm{~dB}$. To enhance the total gain, the approach is to use additional deflection cavities. The gyrocon experiment reported by Balakin, for instance, has a gain of up to $60 \mathrm{~dB}$ with as many as 5 cavities. Karliner's magnicon demonstrated a gain of $25 \mathrm{~dB}$ with two deflection cavities. Here we work out the simplest theory of the amplification by additional cavities.

If the electric field in the $(n-1)$ th cavity is $E_{n-1}$, we would like to calculate the field in the $n$th cavity $E_{n}$. To calculate this, we use linear theory in the bunching cavities. Furthermore, we assume that as the beam traverses the cavity, the angular deflection is calculated as if it traveled along the axis (i.e., (16)). As the beam deflects, it enters the next cavity at an offset position and thereby excites an oscillation in that cavity. Assuming the beam travels on a straight line parallel to the axis (i.e., assuming that the separation between the cavities is long compared to the width of the cavity), the amplitude of the excited mode can be calculated relatively simply.

If the separation between the cavities is $\Lambda$, the displacement of the beam as it enters the next cavity is given by

$$
d=\frac{e h E_{n-1} \Lambda}{\gamma m c \omega}\left[1-\cos \frac{\omega L}{v_{z}}\right] .
$$

To calculate the fields in the $n$th cavity, we use (24). However, for the sake of simplicity here, we do not consider the effect of beam loading of the prebunching cavities. This can be accounted for in a straightforward way by the techniques developed in Section I. For the orbit, we use the unperturbed orbit:

$$
\begin{array}{rlrl}
x\left(t_{n}, t\right) & =x\left(t_{n}\right), & y\left(t_{n}, t\right)=y\left(t_{n}\right) & (50 \mathrm{a}) \\
x\left(t_{n}\right) & =\Lambda \alpha \cos \phi_{n-1}, & y\left(t_{n}\right)=\Lambda \alpha \sin \phi_{n-1} \\
\phi_{n-1} & =\frac{\omega \Lambda}{2 v_{-}}+\omega t_{n-1}+\zeta_{n-1}
\end{array}
$$

where $\phi_{n-1}$ is the azimuthal phase on exiting the cavity. Here we have assumed an arbitrary phase for the wave $\zeta_{n-1}$ to distinguish the fact that the fields in the different cavities could have different phases. In complex notation we denote $F=E \exp i \zeta$. Then (24) can be used to calculate the field in the $n$th cavity. Using (50) for the orbits in (24), we find that

$$
\left\lfloor\frac{\omega\left(\omega+\frac{i k_{t} c}{Q}\right)}{c^{2}}-k_{t}^{2}\right\rfloor F_{n}=D F_{n-1} \exp i\left(\frac{\pi}{2}-\frac{\omega \Lambda}{v_{z}}\right)
$$

where we have again accounted for the cavity $Q$ on the left-hand side. The quantity $D$ is given by

$$
D=\frac{4 \sqrt{\pi} k_{t}^{2} I v_{z} e h \Lambda}{a L J_{1}^{\prime}\left(k_{t} a\right) \gamma m \omega c^{3}}
$$

where we have assumed that $\omega L / v_{z}=\pi$ so as to achieve maximum deflection. For the $\mathrm{TM}_{110}$ cavity which we have been considering, for a $1-\mathrm{MeV}$ beam we find that

$$
D=5.6 \times 10^{-5} I(\mathrm{~A}) \Lambda
$$

where the current $I$ is in amperes and the intercavity separation $\Lambda$ is in centimeters. The maximum gain will occur at the resonant frequency of the cavity (actually, at the frequency corrected by the beam loading which was calculated in Section III-D). The maximum gain is given by

$$
\left|\frac{F_{n}}{F_{n-1}}\right|=\frac{2 D Q c^{2}}{\omega^{2}} \text {. }
$$


For a 100-A beam, with $\Lambda=5 \mathrm{~cm}$ and $Q=400$, the gain is about a factor of 4 in field, or a factor of 16 in power. Thus it appears to be possible to design a magnicon with several deflection cavities in order to increase the maximum gain.

\section{Summary}

To summarize, the previous results on gyrocons and magnicons both here and in the Soviet Union have been reviewed. Each device has the potential of operating at very high average power and efficiency. For the gyrocon, the restriction is that the beam has high voltage and low current. This is necessary both to guide the beam through the drift section and into the output cavity. Once in the output cavity, the interaction is like that in a klystron. The magnicon also requires low voltage and current, but probably is less constrained than the gyrocon because the entrance to the second cavity is through a large hole rather than a narrow slit. Once in the second cavity, the interaction is like that in a phase-locked gyrotron; it has inherent ability to operate at higher frequency than a gyrocon. The theory of the magnicon was worked out, and a conceptual design was presented for a $10-20 \mathrm{GHz}$ magnicon at 50-70 $\mathrm{MW}$ at 50-70\% efficiency.

\section{ACKNOWLEDGMENT}

The author wishes to thank D. Kirkpatrick and S. Gold for a number of useful discussions. Figs. 4 and 5 were supplied by D. Kirkpatrick.

\section{REFERENCES}

[1] M. M. Karliner et al., "The magnicon-An advanced version of the gyrocon," Nuct. Instrum. Methods Phys. Res., vol. A269, pp. 459474,1988

[2] G. I. Budker et al, U.S. Patent 3885 193, 1975.

[3] G. I. Budker et al., "The gyrocon-An efficient relativistic highpower VHF generator, particle accelerators," Part. Accel., vol. 10, pp. 41-60. 1979

[4] Balakin et al., presented at the European Particle Accelerator Conf., Rome, Italy, June 1988: also presented at SLAC.

[5] R. Adler, private communication.

[6] M. A. Allen, "RF power sources," presented at the European Particle Accelerator Conf., Rome, Italy, June 1988.

[7] R. B. Palmer, "Interdependence of parameters for TeV linear colliders." presented at the ICFA Workshop on Low Emittance Beams, Upton, NY, Mar. 1987

[8] NASA Conf. Publication, in Second Beamed Space-Power Workshop, Langley, VA, Feb. 1989

[9] N. Amitay, V. Galindo, and C. Wu. "Theory and analysis of phased array antennas," AT\&T Bell Telephone, 1972

[10] W. Eckhardt, J. G. Small, and F. Chilton. "Decoy discrimination using ground-based high power microwaves," SPIE, vol. 873 , pp. $112-118,1988$.
[11] V. L. Granatstein et al., " Design of gyroklystron amplifiers for driving TeV e $\mathrm{e}^{+}$linear colliders," IEEE Trans. Nucl. Sci., vol. NS-32, pp. 2957-2960, 1985.

[12] P. J. Tallerico and J. E. Rankin, "The gyrocon: A high-efficiency, high power microwave amplifier," IEEE Trans. Electron Devices, vol. ED-26, pp. 1559-1567, 1979

[13] P. J. Tallerico, "Status of the Los Alamos gyrocon," IEEE Trans. Nucl. Sci., vol. NS-28, pp. 2797-2800, 1981.

[14] P. J. Tallerico, "A $150 \mathrm{~kW}, 450 \mathrm{MHz}$ gyrocon RF generator," IEEE Trans. Nucl. Sci., vol. NS-30, pp. 3420-3423, 1983.

[15] P. Tallerico, private communication.

[16] J. Schnurr et al., "Lipid-based tubule, microstructures, thin solid films,"' Thin Solid Films, vol. 152, pp. 181-206, 1987.

[17] D. A. Kirkpatrick and R. Price, to be published.

[18] R. R. Nag, Theory of Electrical Transport in Semiconductors. New York: Pergamon, 1972, chap. 7.

[19] K. Kirkpatrick, Ph.D. dissertation, M.I.T., Cambridge, Jan. 1988.

[20] J. Lawson, Physics of Charged Particle Beams. New York: Oxford Univ. Press, 1977.

[21] K. J. Harker, "Determination of electrode shapes for axially symmetric electron guns," J. Appl. Phys., vol. 31, pp. 2165-2170, 1960.

[22] P. Kirstein, G. Kino, and W. Waters, Space Charge Flow. New York: McGraw-Hill, 1967.

[23] A. W. Fliflet et al. " "Use of electrode synthesis techniques to design MIG-type guns for high power gyrotrons,"' Int. J. Electron., vol. 53, pp. $743-754,1982$.

[24] J. Finn, A. W. Fliflet, and W. M. Manheimer, "One dimensional models for relativistic electron beam diode design," Int. J. Electron., vol. 61 , pp. $985-1005,1986$

[25] A. Ganguly and K. R. Chu, "Limiting current in gyrotrons," Int. J. Infrared and Millimeter Waves, vol. 5, pp. 103-121, 1984.

[26] P. Sprangle, C. Tang, and W. M. Manheimer, "Nonlinear theory of free electron lasers and efficiency enhancement," Phys. Rev., vol. A21, pp. 302-319, 1980.

[27] A. Bondeson, W. Manheimer, and E. Ott "Multimode analysis of quasi-optical gyrotrons and gyroklystrons," in Infrared and Millimeter Waves, K. Button, Ed. New York: Academic, vol. 9, pp. 309-341, 1983.

[28] S. W. MacDonald et al. "Boundary integral method for computing eigenfunctions in slotted gyrotron cavities of arbitrary cross section," Int. J. Electron., vol. 61, pp. 795-822, 1986.

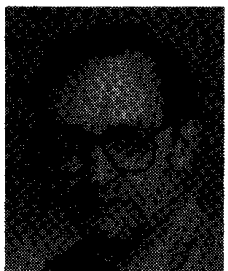

Wallace M. Manheimer (M'59-SM'69) was born in New York City. He received the S.B. degree from the Massachusetts Institute of Technology, Cambridge, (physics), where he also obtained the Ph.D. degree, also in physics.

From 1967 until 1970 he was on the research staff and Physics Department faculty at MIT. From 1970 he has been at the Plasma Physics Division of the Naval Research Laboratory (NRL), Washington, DC. Until 1984 he was in the Plasma Theory Branch and worked in high-altitude nuclear effects, inertial fusion, magnetic fusion, advanced accelerators, and radiation sources. From 1984 to 1989 he was the Head of the High Power Electromagnetic Radiation Branch, which did research in gyrotrons, cyclotron auto resonance masers, and the effect of high-power electromagnetic radiation on military systems. Since July 1989 he has been the Senior Scientist for Fundamental Plasma Processes at NRL. 